\title{
Application of the ISHTAR Suite for the assessment of the health effects of Rome environmental policy
}

\author{
E. Negrenti ${ }^{1}$, A. Agostini ${ }^{1}$, M. Lelli ${ }^{1}$, P. Mudu ${ }^{2}$, A. Parenti ${ }^{3}$ \\ \& C. Lanciano ${ }^{4}$ \\ ${ }^{1}$ ENEA, Italy \\ ${ }^{2}$ WHO-ECEH, Rome, Italy \\ ${ }^{3}$ ASTRAN S.r.l.Viterbo, Italy \\ ${ }^{4}$ G\&O S.r.l.,Rome, Italy
}

\begin{abstract}
The ISHTAR Suite is an innovative software tool that integrates several models for the simulation of the effects of transport and land use policies on the urban environment, population health and artistic heritage. Starting from the simulation of the effects of the postulated measure on the citizens behaviour in terms of daily movements, the suite calculation path goes through the modelling of transport, vehicles safety and emissions of pollutants and noise, pollutants dispersion and noise propagation, exposure to pollutants, noise and accidents and related risk assessment, monuments degradation, up to the overall comparison of the alternative scenarios in terms of cost-benefit or multi criteria analysis. The software modules are integrated by a Suite Manager that controls the tools execution by means of dedicated software 'connectors', and is linked to a User Interface, a suite database and a commercial Geographic Information System. The ISHTAR Suite is now being tested in the seven cities involved in the FP5 EESD Programme ISHTAR Project: Athens, Bologna, Brussels, Graz, Grenoble, Paris and Rome, with the analysis of different measures and policies. In the Rome application the effects on health of a traffic banning policy were studied. Keywords: integrated software, models suite, policies assessment, urban planning, transport systems impacts, traffic banning.
\end{abstract}




\section{Introduction}

Worldwide cities face common challenges concerning their quality of life: degradation of the urban environment, significant risks for citizens health, traffic congestion causing stress and economic inefficiency, progressive damage of the artistic and monumental heritage. Additional difficulties derive from the lack of integrated tools that allow cities to make balanced decisions on a wide range of issues. In this context the European Commission funded in 2001 the ISHTAR Project (Negrenti et al. [1]) aiming at building an Integrated Suite of software models for assessing the impacts of urban policies and actions on the quality of life of citizens, and in particular on traffic congestion, air quality, citizens health and conservation of monuments.

The ISHTAR Project (Negrenti et al. [2]) had several objectives:

- The integration of a large number of software tools and the creation of specific modules for the simulation of key processes such as transport behaviour and its direct impacts on the urban environment.

- The achievement of a high spatial and temporal flexibility in the use of the tool, for maximizing the possibilities of application from local short-term actions to widespread long-term policies.

- Development of specific modelling areas such as the representation of policies effects on citizens behaviour, the 24 hours simulation of traffic emissions, noise and safety, the analysis of air pollution effects on health and monuments.

\section{Methodology}

\subsection{Integration of a relevant number of modelling tools}

The ISHTAR Suite is based on a high number of software tools whose aim is the modelling of various aspects of the impact analysis of short-term actions and long-term policies. Standard models suites normally include only a few of those models. It also represents a strong enlargement of the applicability area, since with this kind of 'multi-impacts' suite the users are able to analyse in an integrated and 'coherent' way the various aspects of 'global' urban policies, without having to perform separate studies relying on different input data. The wide scope of applicability offers the potential of easing the cooperation between different departments in municipal authorities: in fact the tool will be of interest for planning and assessment work in the transport, environment, health and artistic heritage sectors.

\subsection{Evolution of modelling techniques in crucial impact areas}

The scientific core value of the Suite is largely linked to a few crucial modelling developments on which the accuracy and the significance of the results deriving from the Suite application strongly depend. These areas are: 
a) prediction of the effects of citizens' reaction to postulated measures.

b) improvement of the modelling of vehicle emissions, particularly concerning the consideration of speed variability along the network links, and the spatialtemporal distribution of 'cold start emissions'.

c) development of an urban road safety model, which can take into account the variable flow levels in the network

d) disaggregated estimate of pollution effects on citizens' health based on the analysis of population groups' movements.

\subsection{Realisation of integrated specific modules inside the suite}

The ISHTAR Suite aims at the highest flexibility of use: this is reflected in some of the tools. The choice of building an 'Integrated Transport Module' which makes use of different models, having complementary characteristics in terms of applicability field, is of key significance. Also relevant is the consequence of this flexibility in transport modelling: the downstream models (emission, noise, safety, exposure models) will have to be flexible in their input characteristics in order to give the proper accuracy whichever transport model is used. This implies the use of 'advanced' emission, noise and safety models capable of treating flexible input data. Also the module dedicated to the overall evaluation of the policy scenarios includes parallel elements: in this case a Cost Benefit Analysis tool is complemented by a Multi criteria Analysis software.

\subsection{Space and time flexibility}

Among the crucial characteristics of the Suite, a large flexibility in space and time plays an essential role. The starting point for the achievement of this goal was the realization of the so called '24hours capability': traffic flows, vehicles speed, emissions, noise levels, pollution levels are calculated (when needed) hour by hour thanks to the characteristics of the citizens behaviour, transport, emission, noise and dispersion models that have been selected or developed. This flexibility greatly enhances the scope of applicability of the tool.

\section{Project results}

The ISHTAR Suite (Negrenti et al. [3]) was built over the following software modules including one or more software tool. The modules exchange a number of data, schematically represented in Figure 1.

\subsection{The Cellular Transport Methodology}

The Cellular Transport Methodology (CTM) is a new software tool developed by ISIS (Italy) that simulates the effects of policies and measures on the behaviour of citizens in terms of movements, thus producing the modified OriginDestination matrices. This tool is considered as an 'ancillary element' of the suite because it is likely that the city teams wishing to use the ISHTAR software 
will already have a local 'mobility demand model' or alternative techniques for estimating the modification of the trip matrices.

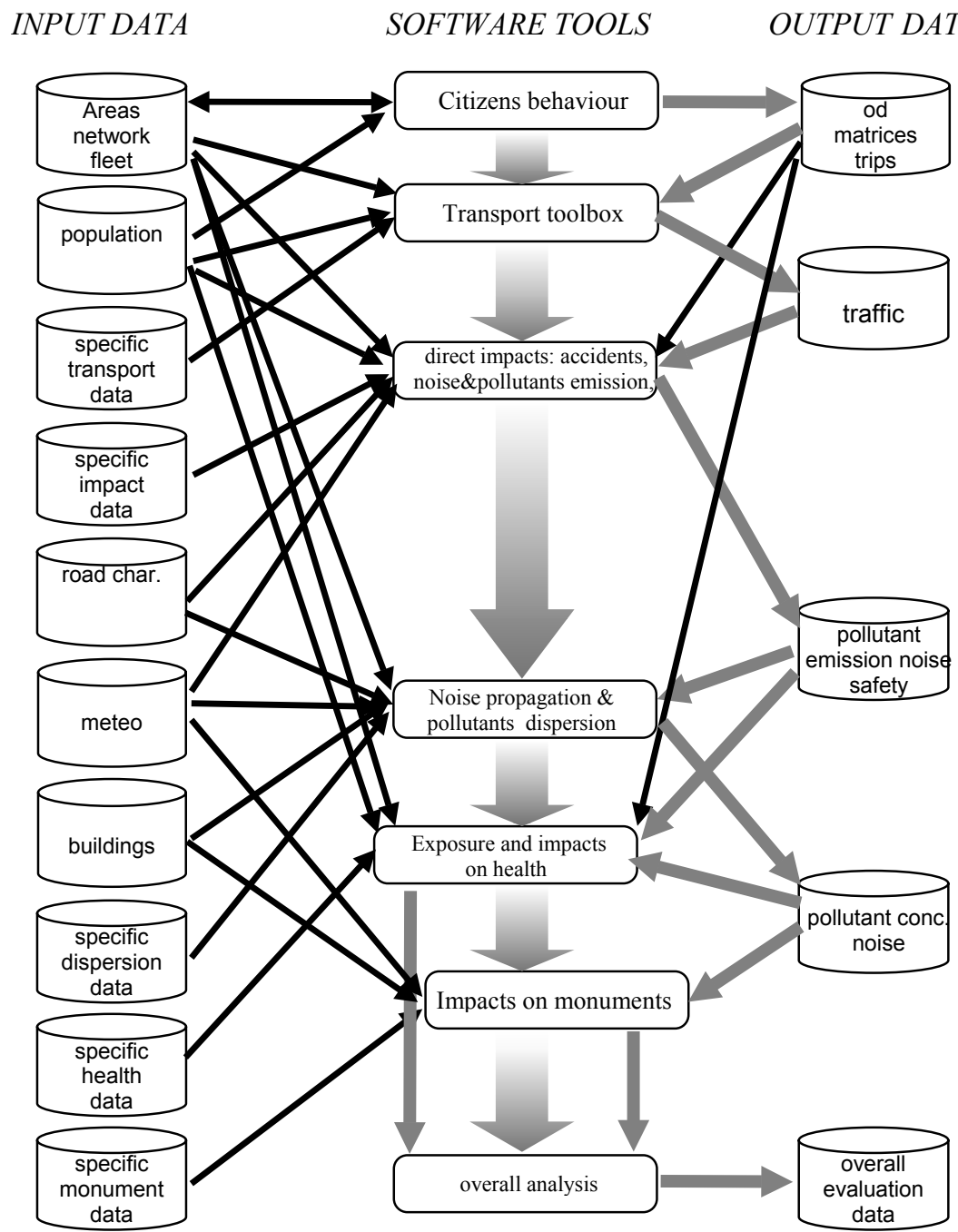

Figure 1: $\quad$ Main Data flows and Input-Output Data in ISHTAR Suite.

\subsection{The transport toolbox}

After an analysis of the available transport models, the new VISUPOLIS model has been selected as the best tool to integrate in the suite. This model has been developed by PTV (Germany) integrating the well-known VISUM model and the innovative tool 'METROPOLIS' by Prof. A. De Palma from the University 
of Clergy Pontoise (F). However the potential users are free to continue to use their own traffic model (as most of the cities participating in ISHTAR Project did). VISUPOLIS is now being tested in the Paris case study (the Car Free day).

\subsection{The Transport Direct impacts module}

The direct impacts model chosen for the suite is TEE2004, developed by ENEA and ASTRAN (Italy). This tool is particularly flexible in terms of space and time, includes advanced modelling of kinematics and cold start effects on the emissions, and feeds several downstream suite elements by calculating the emissions of pollutants and noise and the occurrence of accidents. The tool is compatible with most of the traffic models output. In facts the large number of options about the description of vehicle kinematics, the definition of the local fleet at link level and the approach for estimating the fraction of cold vehicles guarantees an easy coupling with the upstream used traffic model.

\subsection{Noise propagation and pollutants dispersion module}

The pollutants dispersion can be calculated with one of the two tools provided by ARIA Technologies depending on the spatial and time scale. For urban scale and long term analysis the suite will rely on ARIA Impacts, while for meso scale and short term events ARIA Regional will be the future reference, not yet fully integrated in the suite. For the noise propagation the Soundplan software (by Braunstein and Berndt GmbH - D) has been integrated. These software tools operate on a common and harmonised set of input data.

\subsection{Exposure and Impacts on Health module}

For assessing population exposure to pollutants and noise, a completely new software denominated TEX (Transport Exposure) has been developed by WHO (ECEH office in Rome). Such a tool provides exposure of population groups in their residential areas or along the trips in the city network. The evaluation of the health risk related to the exposure to pollutants, noise and accidents is run with the HIT software, also developed by WHO. This tool provides estimates of life years lost due to air pollution, noise annoyance and accidents effects.

\subsection{Impacts on monuments}

The air pollutants impact on monuments is simulated by a software developed by ENEA (I) and PHAOS (GR). This software named MODA (Monuments Damage) can assess the loss of material and the deposition of crust and the money needed for maintenance. The model provides estimates of damage for specific monuments or for types of monuments and buildings.

\subsection{Overall scenarios analysis tool}

For the overall analysis of the policy scenarios two methodologies and software pieces are available: the Cost-Benefit Analysis and the Multi-Criteria Analysis. 
These tools gather the data from the upstream models and give the results of the comparison of the scenarios considered. In any case the MCA takes into account the results of the CBA. Both of them are developed by TRaC - LMU (UK).

\subsection{Software integration}

The integration of the modules is made by a Software Manager that launches the 'software connectors'. The connectors upload the data needed by the single tool in the appropriate format, launches the tool and then downloads the results of the run in the ISHTAR Suite Database making them available for other tools or for the output through the Geographic Information System (ARCGIS) used for managing geographic data. An overall scheme representing the integration architecture of the ISHTAR suite is reported in Figure 2.

\subsection{Case studies}

The suite is being tested with seven case studies involving the cities of ISHTAR project: Athens, Bologna, Brussels, Graz, Grenoble, Paris and Rome. These studies can be summarised as follows.

The Athens case study 'Attiki Odos' addresses the motorway, Attica Periphery Road, which is assessed in terms of traffic, toll strategy and pricing, and environmental impacts. The Bologna Provincial Authority case study concerns the evaluation of infrastructure scenarios for the city of Imola with reference to alternative road paths. The aim of the Belgian case study is to prepare the implementation of traffic banning measures in the Brussels area, according to the Plan Ozone of the Federal Government. In the Brussels case study the focus is on the population behaviour, the modelling of traffic flows and the effects of the measure on pollutant emissions. The Graz case study is based on the traffic and noise impact evaluation of a $600 \mathrm{~m}$ long new road tunnel causing a relevant local traffic rerouting. Grenoble case study is intended to monitor the effects of the installation of reserved lanes for public transportation and new traffic lights on boulevards with heavy traffic. The focus in this case is on traffic and emissions. Every September 22nd the city of Paris takes part in a car free day. This typical short-term event can be modelled with the ISHTAR suite of modules. The results include emissions of pollutants and air pollution. The Rome large-scale case study involves the HEAVEN FP5 Project area banning to the more polluting vehicles. In the northern part of the city centre a number of models of the suite have been used, from the locally available traffic model (Transcad) to the Overall Evaluation module.

\section{Application to the Rome policies for environmental quality}

The city of Rome has established various strategies to tackle traffic problems implementing a framework to rebalance the modal split towards public transport and promote alternative means of transport. An access restriction for non- 
catalysed vehicles has been implemented within the "Rail Ring". The "Rail Ring" area, which surrounds the historic centre, is densely populated with a high concentration of activities making it one of the key areas in the city for interventions to reduce emissions caused by vehicles. This measure had as main effect a change in the fleet composition and no major effects were expected on traffic flows. The measures impact on urban mobility has been performed by the Rome Agency for Mobility (STA) throughout the analysis of detected traffic flows on predefined road links carried out in four different campaigns (November 2001, February 2002, May 2002 and November 2002), one for each stage of the Rail Ring restriction policies implementation.

A simulation of the ISHTAR suite was prepared to test the input-output connections among different software of the suite, data exchange with external software, modelling factors, and clarity of output comprehension. The analysis carried on is based on a comparison between two simulated scenarios:

1) Do nothing scenario;

2) Actual scenario (based on the traffic measures described above).

Pollutants modelled both by the Emission and Dispersion modules were $\mathrm{CO}$ and PM10. CO was also modelled by the exposure module and PM10 by the Health effects module.

\subsection{Area of investigation}

The Rome case study involves the "Heaven" area within the internal rail-ring, excluding the Rome "ZTL" (Limited Traffic zone) that covers part of the historical centre. The laboratory area of ISHTAR Project (see Figure 2) has a surface of 16.35 square kilometres. This area represents a "bridge" between the historical Centre and the peripheral North areas. The socio-economic status of the population is here high and there is a big flow of traffic going through the area, mainly in the roman consular streets Nomentana and Salaria, but also through some primary streets. Green areas in this domain are Villa Borghese and Villa Ada (where there is an air quality monitoring station). The OriginDestination Matrices (O/D) used in the traffic simulation phase, with the static assignment model TRANSCAD, are based on the data obtained by the National Statistical Institute (ISTAT) census. An update, with the aim of defining more realistic traffic values has been carried out by means of 40,000 telephone interviews and this made it possible the evaluation on the daily car and motorcycle O/D matrices. The fleet composition is defined in accordance with ACI (Italian Automobile Club).

Rome air monitoring network consists of 13 monitoring stations classified in four different types: A (located in areas not directly affected by traffic sources such as park or green areas), B (located in areas with heavy traffic conditions), C (located in residential areas) and D (located outside the urban area, almost in the countryside). The monitoring network acquires concentration data every hour. Data are sent to the regional environmental protection Agency (ARPA) for validation and delivered to the Environmental Department of the Municipality of Rome that is responsible for data collecting, storing and delivering. Moreover the 
Municipality of Rome carries on campaigns with diffusion tubes. These campaigns last one week and are distributed on the whole urban area, during different periods of the year. Monitoring tubes are usually located in secondary streets, to give information on background pollution. Population data include:

Registered residents in the area in the year 1999, split by gender and age;

Registered residents in the area in the year 2000;

Working people in the year 1991.

The population registers were used as source of population data

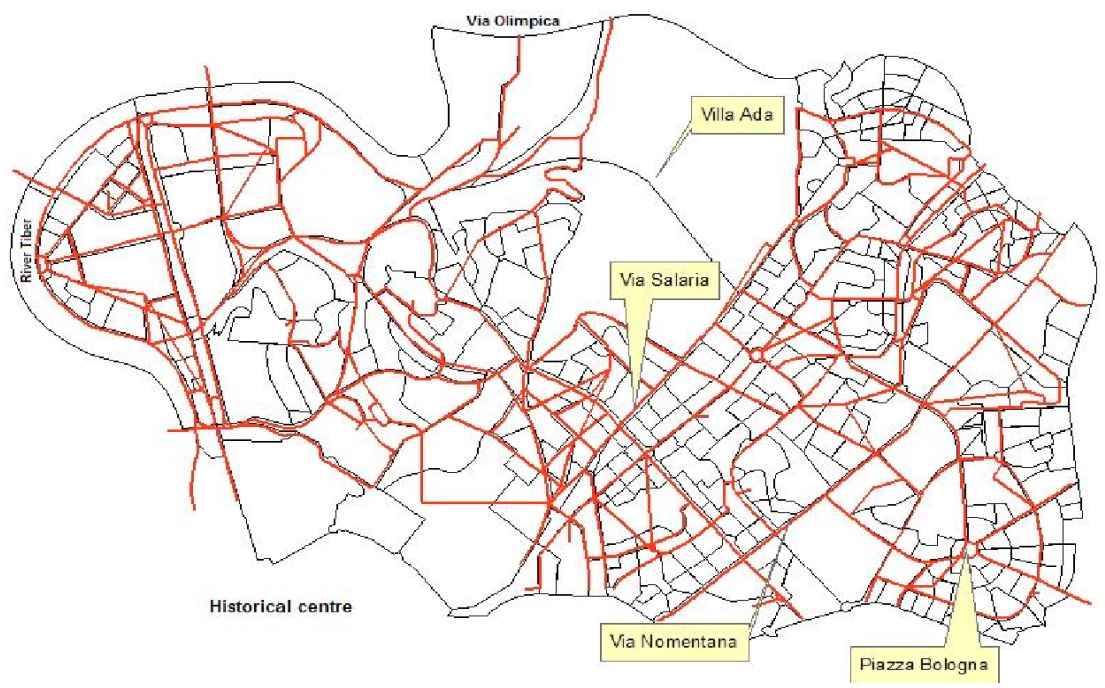

Figure 2: The HEAVEN area considered for the application of the ISHTAR Suite.

\subsection{Impacts of the measure}

Taking into account the traffic data (flows and speeds along all the links) calculated by TRANSCAD, the direct impact model (TEE2004) software has been used for the calculation of CO and PM10 emissions. The aggregated results of TEE during the simulation day shows the reduction of $\mathrm{CO}$ of about $50 \%$, in terms of emissions, confirms the validity of the adoption of the catalyst system regarding such kind of pollution. Regarding the Particulate Matter with diameter below 10 micrometer the emission reduction is about 18 percent. Pollutant concentrations, the 12 th of November 2001, have been calculated for CO and PM10 with the Gaussian dispersion model ARIA IMPACT (by Aria Technologies) taking into account the emission data calculated by TEE, the measured meteorological data, the topography and the background measured by the Villa Ada monitoring station.

The pollutant concentrations due to the emission within the Heaven area are low, as the area is quite small and surrounded by the city, in fact the 
concentration increase due to the calculated emissions coming from the road traffic, compared to the background is about $2,5 \%$ for PM10 and $10 \%$ for CO. In any case the difference in terms of concentration between the two scenarios is $0,33 \%$ for PM10 and $4,76 \%$ for CO.

After running the ARIA model in ISHTAR it is possible to calculate the exposure of people to a pollutant through software TEX (Traffic EXposure). The dispersion values considered for an exposure assessment cover the period of one day (the model refers to the 12th November 2001). The data, obtained from a Transcad output produced by STA, were considered as an hourly series from midnight to midnight. As expected, results indicate a stronger exposure of the people living and working on the main arterial network but also some area of high exposure of $\mathrm{CO}$ with low population concentration and some areas with lower level of exposure with high population concentrations (see Fig.3). While the exposure output, if all inputs are available, offer an evaluation of the relation between a spatial dispersion of a pollutant and the distribution of the population, the application of dose-response curves happens at a broader approximate level. In fact, as it is well-known to assess the effects of a particular pollutant, in our case of ambient PM10 pollution, over an exposed population, there are two assumptions to be done: the concentration consists of the average measured in the city or area where the population live, everyone is assumed to be exposed to the same average concentration. With this information it is possible to calculate the attributable risk as a proportion, while to obtain the absolute number of attributable cases it is necessary to know, in addition, the observed rates of disease or mortality occurrence in the population under study. Within the ISHTAR suite the HIT software is included to assess the impacts of different sources of traffic-generated pollutants.

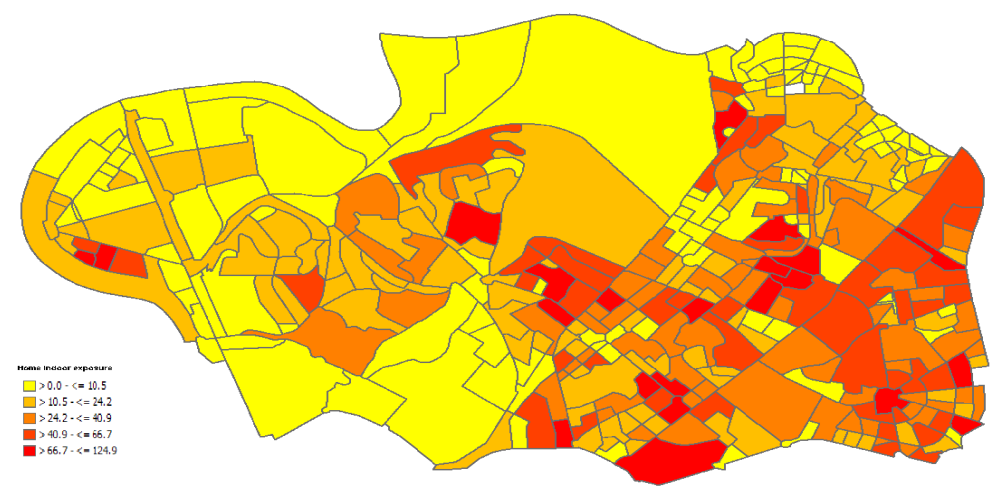

Figure 3: Exposure simulation output.

The estimation of health impacts is possible by applying dose-response curves made available through the analysis of available literature. In the case under investigation the impact was simulated of the average value estimated for PM10 
as it was a yearly average affecting the whole resident population. Total mortality was chosen as health end-point, both for the 'do nothing' and 'actual' scenarios. Concentrations of PM10 greater than 20 micrograms per cubic meter, considering an average of 30 produces a long term effect in terms of estimated number of excess cases of $337(95 \% \mathrm{CI}=110-558)$ and short term effects as 27 $(95 \%$ CI $=23-32)$. Estimates are not only intended as "impact" on health, but ideally as "gains" that would be achieved by reducing average concentrations.

\section{Conclusions}

The ISHTAR Suite has the potential of becoming a reference tool for the future planning of urban policies in terms of traffic, environment, health, monuments protection (Negrenti [4]). The integrated tool will ease cooperation among planning departments of municipalities and will be of interest also for environmental agencies, consultants, transport companies, ministries. Project conclusion in May 2005 will be followed by a pre-commercialisation phase allowing the involved partners to transform a research project result into a marketable tool or a software system for running calculation services. Future users of the suite are expected in a fully international environment, having assessed the high interest by audiences worldwide (Negrenti [5] and Negrenti and Agostini [6]).

\section{References}

[1] Negrenti, E. et al.: ISHTAR Project Proposal to EC DG RES (Issued by ENEA as Project Coordinator), p. 1-99, 2000.

[2] Negrenti, E. et al.: ISHTAR Contract EVK4 CT-00034 (issued in Brussels by EC DG RES) p. 1-xx , 2001.

[3] Negrenti E. et al.: ISHTAR web site: http://www.ishtar-fp5-eu.com, 2002.

[4] Negrenti, E and Hoglund P. 'ISHTAR: an Integrated Models Suite for Sustainable Regional and Town Planning - Cities of Tomorrow Conference - Goteborg (S) - 23-24 August 2001.

[5] Negrenti, E. 'ISHTAR Project: Building a Model Suite for Urban Sustainability - 21st ARRB/11th REAAA Conference 'TRANSPORT our highway to a sustainable future' - Cairns - 18-23 May 2003.

[6] Negrenti, E. Agostini, A. 'ISHTAR': 'integrated software for health, transport efficiency and artistic heritage recovery' 'Transport induced Air Pollution conference - Boulder (CO), September 2004. 